Med Klin Intensivmed Notfmed 2019·114:192-193 https://doi.org/10.1007/s00063-019-0558-7 Online publiziert: 11. März 2019

(c) Springer Medizin Verlag GmbH, ein Teil von Springer Nature 2019

\section{U. Janssens ${ }^{1} \cdot W$. Druml ${ }^{2}$}

${ }^{1}$ Klinik für Innere Medizin und Internistische Intensivmedizin, Sankt-Antonis-Hospital Eschweiler, Eschweiler, Deutschland

${ }^{2}$ Klinik für Innere Medizin III, Abteilung für Nephrologie, Allgemeines Krankenhaus Wien, Wien, Österreich

\title{
Übertherapie in der Intensivmedizin
}

Im Jahr 2017 erschien im The Lancet eine beachtenswerte Serie unter dem Titel „Right Care“ $[1-3,5]$. Einer dieser Beiträge widmete sich dem weltweiten übermäßigen Einsatz medizinischer Leistungen („overuse of medical services“; [1]). Dabei wird klar, dass eine Übertherapie schwierig zu messen und auch nicht genau definiert ist. Übertherapie bzw. der übermäßige Einsatz medizinischer Leistungen (Screening, Diagnostik, Medikamente, Prozeduren etc.) kann im weitesten Sinne als Gesundheitsversorgung oder Behandlung definiert werden, die nicht zu einer nennenswerten Verbesserung der Lebensqualität und -quantität führt, mehr Schaden als Benefit verursacht oder von Patienten, die umfänglich über Nutzen und Schaden informiert wurden, nicht gewollt wurde [1]. Übertherapie führt zu körperlichen, psychischen und sogar finanziellen Belastungen bzw. Schäden bei den betroffenen Patienten, lenkt in nicht unerheblichem Ausmaß die Ressourcen im Gesundheitssystem und Sozialausgaben um und wird in nahezu allen Bereichen der Medizin beobachtet [1].

Das Risiko einer Überversorgung bzw. Übertherapie ist in der Intensivmedizin der Industrienationen, aber v. a. in Deutschland außerordentlich hoch. Die technischen Möglichkeiten haben sich hier in den letzten Jahren rasant weiterentwickelt. Beispielhaft seien extrakorporale Herz-Kreislauf-Unterstützungssysteme genannt, die mittlerweile nahezu flächendeckend zum Einsatz kommen, obwohl robuste Daten randomisiert kontrollierter Studien zur Effektivität in Bezug auf harte Endpunkte bei den allermeisten Krankheitsbildern fehlen. Ebenfalls beobachten wir in Deutschland seit mehreren Jahren eine exponentielle Zunahme außerklinischer Beatmungsfälle, die neben den erheblichen ökonomischen Auswirkungen auch ethische Fragen aufwerfen [4].

Dieser Themenschwerpunkt in der Zeitschrift Medizinische Klinik - Intensivmedizin und Notfallmedizin wurde konzipiert, um aus verschiedenen Blickwinkeln die Aufmerksamkeit der Leserinnen und Leser auf dieses brisante Thema „Übertherapie in der Intensivmedizin“ zu lenken. In einem Übersichtsbeitrag gehen W. Druml et al. (https://doi. org/10.1007/s00063-019-0548-9) auf die verschiedenen Aspekte der Übertherapie in der Intensivmedizin ein und betonen, dass alle Bereiche der Intensivmedizin betroffen sind. Gerade am Lebensende wird immer wieder der Einsatz einer sinnlosen Therapie beobachtet. Auch in der Intensivpflege tritt das Phänomene einer „Überpflege“ in der Praxis tatsächlich häufig auf, wird aber selten in der Literatur berichtet. Der Beitrag von M. Wohlmannstetter (https://doi. org/10.1007/s00063-019-0530-6) belegt, dass auch Überpflege konkrete, negative Auswirkungen auf den Zustand von Patienten haben kann. Eindringlich weisen C. Karagiannidis et al. (https://doi.org/10. 1007/s00063-019-0533-3) auf die Probleme der extrakorporalen Lungen- und Herz-Lungen-Ersatzverfahren (ECMO) hin: Hier besteht immer wieder eine Diskrepanz zwischen der technischen Machbarkeit und einer patientenzentrierten Therapie, die sich sehr streng an ethischen Grundprinzipien ausrichten sollte. Auch hämatologisch-onkologische Patienten werden zu Recht immer häufiger auf einer Intensivstation behandelt. Ausdrücklich betonen $M$. Kochanek et al. (https://doi.org/10.1007/s00063019-0532-4) dass wie bei allen Patienten auch in diesem Kollektiv eine klare ärztliche Indikation die Voraussetzung für eine intensivmedizinische Behandlungsoption darstellt und gleichzeitig auch die Grenzen einer solchen Intensivtherapie im Vorfeld gemeinsam mit den Patienten und Angehörigen ausgelotet werden müssen. Übertherapie ist nicht nur für Patienten eine Belastung und kann Schaden verursachen - auch im Behandlungsteam kann die Wahrnehmung einer Übertherapie zu Burn-out bis hin zur Kündigung und bei Angehörigen $\mathrm{zu}$ erheblichen Langzeitfolgen mit Angst und Depression führen, wie D. Schwarzkopf (https://doi.org/10.1007/ s00063-019-0531-5) in seinem Beitrag eindrucksvoll darstellt. Last, but not least verweist G. Duttge (https://doi.org/10. 1007/s00063-019-0545-z) auf die relevanten juristischen Implikationen einer Übertherapie und gibt den Ratschlag, das klinische Feld systematisch nach typischen Anwendungsfällen „nutzloser“ bzw. „unangemessener“ Behandlungsmaßnahmen zu sondieren.

Es ist gelungen, ein hochaktuelles und spannendes Themenheft zusammenzustellen, das Sie, die Leserinnen und Leser, nicht nur in den Bann ziehen, sondern Ihnen sicherlich Anlass geben wird, im klinischen Alltag noch mehr als bisher auf eine Übertherapie zu achten. Nur das Erkennen und Benennen einer Übertherapie wird dazu führen, dass Belastung und Schaden von Patienten, Angehörigen aber auch dem Behandlungsteam abgewendet wird. 
Wir dürfen uns bei den Autoren ganz ausdrücklich für die exzellenten Beiträge bedanken und wünschen den Leserinnen und Lesern eine aufschlussreiche Lektüre!

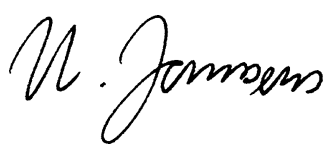

Uwe Janssens, Eschweiler<smiles>CCCCCCCCCCCCCCC(C)C</smiles>

Wilfred Druml, Wien

\section{Korrespondenzadresse}

Prof. Dr. U. Janssens
Klinik für Innere Medizin
und Internistische
Intensivmedizin, Sankt-
$\begin{aligned} & \text { Antonis-Hospital Eschweiler } \\ & \text { Dechant-Deckers Straße 8, } \\ & 52249 \text { Eschweiler, } \\ & \text { Deutschland } \\ & \text { uwe.janssens@ } \\ & \text { sah-eschweiler.de }\end{aligned}$

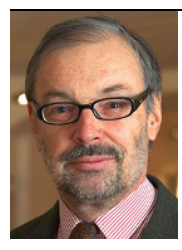

Prof. Dr. W. Druml

Klinik für Innere Medizin III, Abteilung für Nephrologie, Allgemeines Krankenhaus Wien

Währinger Gürtel 18-20, 1090 Wien, Österreich wilfred.druml@ meduniwien.ac.at

Interessenkonflikt. U. Janssens und W. Druml geben an, dass kein Interessenkonflikt besteht.

\section{Literatur}

1. Brownlee S, Chalkidou K, Doust J et al (2017) Evidence for overuse of medical services around the world. Lancet 390:156-168

2. Elshaug AG, Rosenthal MB, Lavis JN et al (2017) Levers for addressing medical underuse and overuse: achieving high-value health care. Lancet 390:191-202

3. Glasziou P, Straus S, Brownlee S et al (2017) Evidence for underuse of effective medical services around the world. Lancet 390:169-177

4. Karagiannidis C, Strassmann S, Callegari J et al (2018) Epidemiologische Entwicklung der ausserklinischen Beatmung: Eine rasant zunehmende Herausforderung fur die ambulante und stationare Patientenversorgung. Dtsch Med Wochenschr. https://doi.org/10.1055/a-0758-4512

5. Saini V, Garcia-Armesto S, Klemperer D et al (2017) Drivers of poor medical care. Lancet 390:178-190

\section{Deichert, Ulrich, Höppner, Wolfgang, Steller, Joachim (Hrsg.) Traumjob oder Albtraum? Chefarzt m/w}

Ein Rat- und Perspektivgeber

Berlin Heidelberg: Springer-Verlag 2016, 1. Auflage, 640 S., (ISBN: 978-3-66249778-4), Hardcover 49,99 EUR

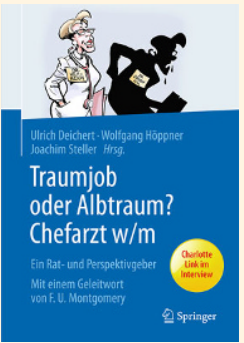

Parallel zur nachhaltigen Veränderung unseres Gesundheitswesens in den vergangenen drei Dekaden hat sich das Berufsbild des Chefarztes gewandelt. Was zunächst in einzelnen Berichten über ausgeprägte Differenzen zwischen Anspruch und Wirklichkeit im Chefarztdasein begann, hat sich inzwischen sowohl in fokussierten soziologischen Untersuchungen, als auch im allgemeinen Bewusstsein der Fachkollegen verdichtet: Im historischen Vergleich zur vorausgegangenen Generation sind heutige leitende Ärzte konfrontiert mit immensen Rollenkonflikten zwischen dem Einsatz für das Patientenwohl, ökonomischen Ansprüchen zur Gewinnmaximierung der Krankenhäuser, Grenzsetzungen ihrer Mitarbeiter hinsichtlich der Arbeitszeiten bei gleichzeitig vermehrter Einforderung einer qualitativ hochwerten Weiterbildung, allseitiger Transparenz und Qualitätskontrolle neben dem eigenen Anspruch auf medizinische Exzellenz und ständig steigender Erwartungshaltung der Patienten und Angehörigen auch über das unmittelbar Medizinische hinaus. Hinzu kommt eine ansteigende Klagebereitschaft der Behandelten. Die Gehälter der Chefärzte sind gesunken, das soziale Ansehen ist zumindest angesichts eines ständigen Bedarfs nach Skandalisierung in den Medien zahlreichen Angriffen ausgesetzt.

Unter diesen Eindrücken bedient das Buch einen großen Bedarf nach sachlicher Systematisierung. Sehr umfassend werden aus allen denkbaren Perspektiven mit viel Informationsgehalt sowohl die historischen Hintergründe, das ökonomische Umfeld, juristisches Fachwissen und zahlreiche Fallstricke für die Tätigkeit des heutigen Chefarztes aufgezeigt. Das Spannungsfeld zwischen Ethik und Ökonomie wird differenziert analysiert. Hierzu kommen jeweils erfahrene und exponierte Fachleute in jedem Kapitel zu Wort. Die Lektüre eignet sich ganz entsprechend dem Titel des Buches sowohl für jeden, der über den Karriereschritt in diese veränderte Welt nachdenkt - und vielleicht zögert - , als auch für bereits Betroffene als anregende und umfangreiche Quelle und Wegweiser. Wer die Spannungen im Umfeld dieser beruflichen Funktion selber erlebt oder am Rande erspürt, liest dieses Buch mit großem Erkenntnisgewinn und Anregung zur Selbstreflexion.

Gleichzeitig gelingt es den Herausgebern durch die geschickte Auswahl der sehr differenzierten Darstellungen in den einzelnen Kapiteln, die abschließende Bewertung der im Titel gestellten Frage „Traumjob oder Albtraum?" offen zu lassen. Der Leser hat das Vergnügen, sich selbst zu entscheiden. So viel aber sei verraten: das Buch macht durchweg Lust, sich der Herausforderung zu stellen und gibt wertvolle Anregungen zur kreativen Ausgestaltung des offenbar vollkommen gewandelten Berufsbildes Chefarzt m/w.

So liegt hier ein absolut empfehlenswertes Buch für eine breite Leserschaft vor.

Dr. med. Erik Allemeyer, Münster 